\title{
Resolución de problemas en química: descifrando métodos, errores, obstáculos, temáticas y aplicabilidad usando N-Vivo
}

\author{
Sulma P. Vera-Monroy', Verónica De la $\mathrm{Hoz}^{2}$, y María C. Gamboa ${ }^{3^{*}}$ \\ (1) Facultad de Ingeniería, Grupo de Investigación CAPSAB, Universidad de La Sabana, Colombia \\ (correo-e: sulmavemo@unisabana.edu.co). \\ (2) Facultad de Ciencias de la Educación, Programa de Licenciatura de Educación Especial. Grupo de investigación \\ Enl@ce, Universidad del Atlántico. Puerto Colombia- Atlántico (correo-e: veronicadelahoz@mail.uniatlantico.edu.co). \\ (3) Escuela Ciencias de la Educación. Universidad Nacional Abierta y a Distancia UNAD. Grupo de Investigación \\ Ambientes de Enseñanza Aprendizaje de las Ciencias Básicas y Sociales - AMECI. Bogotá, Colombia (correo-e: \\ maria.gamboa@unad.edu.co).
}

* Autor a quien debe ser enviada la correspondencia.

Recibido Jul. 19, 2021; Aceptado Sep. 23, 2021; Versión final Oct. 27, 2021, Publicado Feb. 2022

\begin{abstract}
Resumen
El alcance de esta investigación fue identificar los métodos, los errores y los obstáculos reconocidos por los estudiantes, y las temáticas y la aplicabilidad que los profesores le otorgan a la resolución de problemas en química. Participaron 9 profesores y 388 estudiantes de cinco universidades colombianas y una argentina. La investigación fue de enfoque cualitativo y se implementó el análisis narrativo como metodología bajo un paradigma hermenéutico-interpretativo, empleando la técnica de grupos focales. Se establecieron categorías y los datos cualitativos fueron procesados con el software NVivo. Los resultados revelaron que con respecto a los errores y obstáculos, los más predominantes corresponden a la omisión de algunas unidades, el inadecuado manejo de las equivalencias y el establecimiento de relaciones entre unidades. Se concluye que el análisis dimensional es el método más útil para solucionar correctamente problemas químicos que involucran lógica matemática como propiedades, soluciones, estequiometría y gases.
\end{abstract}

\section{Problem solving in chemistry: deciphering methods, errors, barriers, subject matters, and applicability by using N-Vivo} Abstract

The main goal of this research study is to identify student methods, errors, and barriers and to examine the subject matters and applicability that professors give to problem solving in chemistry. Nine professors and 388 students from five Colombian universities and one Argentine university participate in the study. A qualitative research approach is conducted by applying a narrative analysis methodology under a hermeneuticalinterpretive paradigm using the focus group technique. Categories are established and qualitative data are processed on the software NVivo. The results revealed that with regard to errors and barriers, the most prevalent are omission of some units, inadequate handling of equivalences, and improper establishment of relationships between units. It is concluded that dimensional analysis is the most useful method to correctly solve chemical problems that involve mathematical logic such as properties, solutions, stoichiometry and gases. 


\section{INTRODUCCIÓN}

Resolver problemas es un ejercicio que se ha realizado desde el principio de los tiempos, la evolución de la especie humana requirió de acciones específicas que garantizaran la supervivencia y adaptación a nuevos entornos, acciones reconocidas como solucionar problemas; problemas sociales, geográficos, ambientales, físicos, matemáticos, biológicos, clínicos, entre muchos. Todos son problemas que deben seguir una dinámica estructurada para lograr su solución. Al respecto, Bados y García (2014) señalan que el concepto de resolución de problemas se ha abordado desde diferentes perspectivas y ha evolucionado con el tiempo, puede entenderse como el entrenamiento para lograr metas relacionadas con la solución de casos específicos, buscando el desarrollo de habilidades para replicar en futuros problemas.

Díaz y Díaz en 2018 propusieron la heurística para la resolución de problemas, entendido como el procedimiento consciente, planificado y científico de reglas, procedimientos y principios para la exploración y búsqueda de la solución final, propiciando descubrimientos, suposiciones, hipótesis y reglas que movilizan la actividad mental. Resolver problemas es el proceso mediante el cual la situación incierta es clarificada y para ello, se requiere la aplicación de conocimientos y procedimientos específicos, es una actividad compleja que requiere el uso de habilidades de pensamiento de alto nivel.

Los procesos cognitivos involucrados en la resolución tienen que ver con la comprensión, la elaboración, la ejecución y la evaluación de un plan, los cuales llevan a la conciencia metacognitiva de quien resuelve con base en sus conocimientos, la identificación de las variables que intervienen en el problema, la representación adecuada de información y la conciencia sobre qué falta para resolver. Cognitivamente, muchos autores han descrito la resolución de problemas como un procedimiento que reúne tareas extremadamente diversas, reconociendo un problema como una situación prevista o espontánea que produce un cierto grado de incertidumbre y una conducta que busca una solución (Gürsan y Yazgan, 2020; Simamora y Hasratuddin, 2019).

Complementando, Malaspina (2007) identificó la intuición como el primer mecanismo cognitivo que utilizan las personas para resolver problemas, aunado a la habilidad de proponer conjeturas y de formalizar resultados con rigor matemático, el problema radica en el hecho de que en muchos casos la intuición no se acopla a otros procesos que respaldan la rigurosidad matemática y finaliza con resultados erróneos. Para Dossey (2017) y Ergen (2020) la resolución de problemas y las matemáticas son dos fenómenos estrechamente entrelazados.

Por otro lado, la regla de tres es conocida como tal, porque existen tres números relacionados en donde se busca calcular el valor de un cuarto dato (Gómez, 2006), es un ejercicio de proporcionalidad que generalmente en las escuelas se aprende de forma directa, siendo un procedimiento básico que depende de la habilidad para relacionar variables e información, el problema es que existen proporcionalidades indirectas que no se ajustan al modelo típico de regla de tres, siendo un método insuficiente o inadecuado para resolver muchos ejercicios matemáticos (Mochón, 2012). En palabras de Morice-Singh (2018) la regla de tres es un razonamiento de proporcionalidad que utiliza el método unitario.

A diferencia de lo anterior, los factores de conversión son equivalencias numéricas que permiten convertir o cambiar los valores de un sistema a otro, organizados en forma de fracciones que permite disponerlos de tal manera que se ajusten a las necesidades del cálculo, es importante aclarar que los factores de conversión por sí solos no resuelven el problema, es preciso reconocer factores implícitos de las situaciones que faciliten el proceso y lleven a buen término la solución (Gamboa et al., 2020). No obstante, el análisis dimensional es el método completo que permite hacer relaciones de proporcionalidad y equivalencia de forma ordenada y sistematizada, organizando la información, las variables y los datos, de tal manera que se evalúe paso a paso las dimensiones del problema, gracias a la disposición algebraica de los diferentes valores con sus unidades en la herramienta matemática, convirtiéndose en una estrategia útil cuando un problema involucra muchas variables porque reduce el conjunto de parámetros y facilita la comparación entre datos aumentando la precisión (Lira-Parada et al., 2021).

Por último, Frodyma en 2020, demostró que el análisis dimensional ayuda a la realización de cálculos complejos en áreas como la fisicoquímica facilitando la determinación de constantes críticas y Bois et al., (2021) lo implementaron para la definición de un modelo para el proceso de formación de espuma de tensioactivos. Adicionalmente, la resolución de problemas en el área de química puede explorarse desde dos enfoques: 1. Aplicando conceptos como el átomo y sus propiedades a nivel molecular, o la periodicidad química y sus características usadas en la mejora de los materiales, entre muchos. 2. Utilizando leyes o teorías relacionadas con la materia, como los estados de agregación, para realizar cálculos determinando magnitudes. De estos últimos, se reconocen temáticas específicas como: estequiometría, soluciones, termodinámica, ácidos, bases, cinética, propiedades coligativas y gases, lo anterior reforzado por Raviolo et al., (2000). 
Por otro lado, Bucheró y Planche-Jardínes en 2020, describieron una lista de errores u obstáculos comunes durante el aprendizaje de la química, entre ellos: 1. Carencia en el uso de procedimientos químicos y metodológicos, 2. Poca ejercitación en la comprensión de los contextos, 3. No saber la forma en la que se resuelven las operaciones, 4. Poco dominio de sistemas y operaciones matemáticas y 5 . Formación en problemas sin contexto ni aplicación. De forma semejante, Despuy et al., (2018) reconocieron que los estudiantes durante su formación identifican errores que están directamente relacionados con la matemática, comprendiendo que cuando tienen dificultades con la matemática básica, también tienen inconvenientes con la resolución de problemas en química. Ellos publicaron una tabla comparativa entre las temáticas de química versus las falencias matemáticas que presentan, así: equilibrio químico - uso de la calculadora (logaritmo); estequiometría y soluciones - uso de regla de tres, proporciones y ecuaciones de primer grado; cinética química - cálculo de recta y pendiente de la recta; leyes de los gases - ecuaciones, proporciones y funciones; y concentraciones y soluciones - conversiones y notación científica. Para evidenciar cómo se resuelven actualmente, problemas en el área de química a nivel universitario, la pregunta de investigación se circunscribe como: ¿Cuál es la percepción que tienen estudiantes y profesores sobre la resolución de problemas en química en relación con los métodos, los errores, los obstáculos, las temáticas y la aplicabilidad?

Finalmente, una herramienta útil para el análisis de las narrativas de los profesores y estudiantes de química en un estudio de corte cualitativo, es NVivo, que permite relacionar datos textuales para la realización de matrices, codificar grandes nodos, y a partir de allí presentar diferentes formas de visualización y exploración gráfica de la información, entre ellas: nubes de palabras, gráficas de barras, diagramas de jerarquías, mapas mentales, y sociogramas, entre otros, facilitando el análisis cualitativo, mucho más organizado y efectivo. El objetivo de este estudio es identificar las percepciones que tienen estudiantes y profesores para resolver problemas en química en cinco universidades colombianas y una argentina, descifrando los métodos, los errores, los obstáculos, las temáticas y la aplicabilidad, a través del análisis de las narrativas y usando la herramienta NVivo (N-Vivo, 2020).

\section{METODOLOGÍA}

El enfoque utilizado es la investigación cualitativa, la cual produce hallazgos a los que no se llega por medio de procedimientos estadísticos u otros medios de cuantificación. Puede tratarse de investigaciones sobre la vida de la gente, percepciones, las experiencias vividas, los comportamientos, emociones y sentimientos, así como el funcionamiento organizacional, los movimientos sociales, los fenómenos culturales y educativos (Corbin y Strauss, 2014). Es una actividad sistemática, de carácter interpretativo, constructivista y naturalista que incluye diversas posturas epistemológicas y teóricas orientadas a la comprensión de la realidad estudiada y/o a su transformación y desarrollo de un cuerpo organizado de conocimientos (Nizama y Nizama, 2020).

La presente investigación se desarrolló bajo el paradigma hermenéutico-interpretativo, el cual, de acuerdo con Morgan (2019), pretende comprender la realidad conservando un pleno sentido de la cultura y sus peculiaridades dentro del fenómeno educativo; es decir, este paradigma surge de la necesidad de describir contextualmente un fenómeno o situación, con el fin de conocer, comprender y actuar. En esta investigación se busca reconocer la percepción que tienen estudiantes y profesores con relación a los métodos, los errores, los obstáculos, las temáticas y la aplicabilidad para la resolución de problemas, se implementa un análisis narrativo como metodología. Según Hernández et. al (2014) se considera que es de carácter descriptivo transversal, porque la recolección de la información se hizo en un solo momento a través de la herramienta de grupos focales (Castro y Miranda, 2019), que de acuerdo con Hernández y González (2020), se convierte en una estrategia grupal de análisis de un tema o problema de interés.

\section{Contexto y Población}

La muestra intencionada se configuró con un total de 388 estudiantes de primer semestre que se encontraban cursando la asignatura de química en el período académico 2020 - 1, de las Universidades Nacional Abierta y a Distancia (UNAD), del Atlántico (UA), Distrital Francisco José de Caldas (UDFJC), La Sabana (UNISABANA) y la de Cundinamarca (UdeC). Adicionalmente este trabajo incluyó 9 profesores del área de química de las universidades UNAD, UA, UdeC, UNISABANA, y Universidad Nacional de la Patagonia Austral (UNPA).

El análisis narrativo se sustenta definiendo unas categorías bases y otras como emergentes que surgieron de la percepción de los actores de investigación, los cuales fueron procesados con el software N-Vivo, el cual apoyó la calificación y la codificación de los mismos. Así mismo, contabilizó las palabras registradas en la base de datos, asistiendo la organización, de tal forma que se establecieron las conexiones entre ellas, arrojando un resumen a través de diversos gráficos, entre los que se destacan: nube de palabras, diagramas de comprensión, gráficas de barras, red semántica y análisis de proporción. 
Para el análisis de los datos las categorías base de los métodos para resolver problemas en química, fueron: el análisis dimensional, la intuición y reglas de tres. En lo referente a los errores en la resolución, se definieron: el mal uso de la calculadora, inadecuado manejo de las equivalencias de las unidades y escribir dos unidades en un término de la fracción. En el caso de los obstáculos, las categorías emergieron de la percepción de los estudiantes. Por último, en lo concerniente a la percepción de los profesores, se pudo analizar dentro de las narrativas, las temáticas y aplicabilidad del análisis dimensional, categorizando los tópicos sobresalientes como: las propiedades de la materia, los gases y la estequiometría y en lo referente a la aplicabilidad del análisis dimensional, las categorías base señaladas fueron: hacer cálculos para resolver problemas, agilizar la capacidad de análisis y evidenciar la relación entre las unidades.

\section{RESULTADOS Y DISCUSIÓN}

A continuación, se muestran los resultados obtenidos en el estudio y su respectiva discusión, presentando de manera separada las declaraciones hechas por estudiantes y profesores.

\section{Métodos utilizados para resolver problemas - percepción de los estudiantes}

En la figura 1, se presenta el análisis de las narrativas y la proporción que genera N-Vivo al contabilizar la frecuencia de las categorías base para resolver problemas, la mayor frecuencia 206 se obtuvo para factores de conversión, seguido por regla de tres (128), intuición (45). Como categorías emergentes se descifraron: no utiliza, con una frecuencia de 2 y uso de videos de YouTube a manera de tutoriales, simuladores en línea, consulta de libros con frecuencia igual a 1.

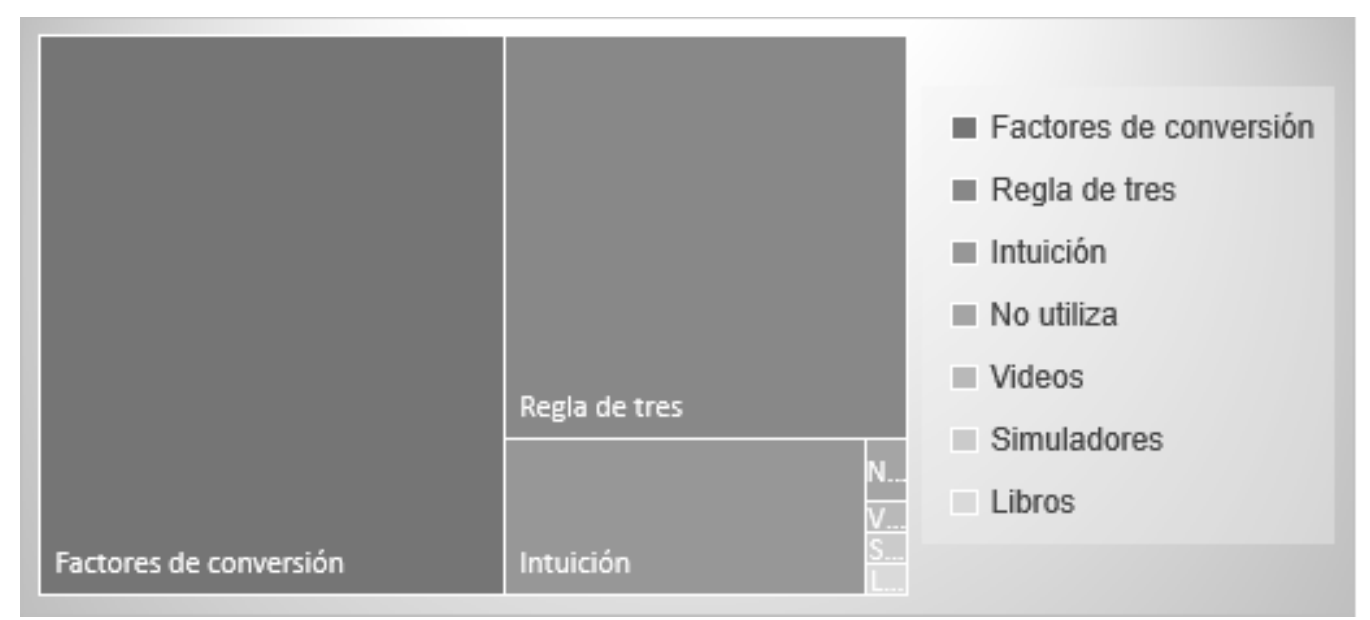

Fig. 1: Métodos usados para resolver problemas

Los resultados muestran que los estudiantes de primer semestre prefieren resolver problemas usando factores de conversión lo que representa hacer análisis dimensional, coincidiendo con los estudios de Anilan \& Berber (2019) quienes demostraron que la resolución de problemas para estudiantes de primeros semestres en licenciatura de física se facilita con el uso de factores de conversión. Adicionalmente, en un estudio de caso sobre una problemática ambiental, tres grupos de estudiantes de las generaciones Millennials y Centennials priorizaron el uso de análisis dimensional para resolver problemas relacionados con lógica matemática (Gamboa et. al, 2020). Sin embargo, una gran porción de estudiantes utiliza la regla de tres, si bien es una estrategia válida, autores como Lira-Parada, et al., (2021) insisten que es un procedimiento que limita el desarrollo de ejercicios que requiere cálculos simultáneos. En cuanto a las categorías emergentes se resalta que los estudiantes reconocen el uso de la tecnología y las nuevas formas de comunicación como métodos para resolver problemas.

\section{Errores y obstáculos más frecuentes en la resolución de problemas - percepción de los estudiantes}

La red semántica que se configuró producto de las narrativas hechas por los estudiantes, visibiliza las acciones que ellos reconocen como las que los conduce a errar durante la resolución de problemas, en orden de relevancia, son: omisión de algunas unidades (131), inadecuado manejo de las equivalencias (128), relación entre unidades (80), mal uso de la calculadora (76), desconocimiento de reglas básicas de las matemáticas (51), no cometo errores (48), escribir dos unidades en un término de la fracción (24); acelerarme, a veces simplemente copio mal los datos, confusión con las fórmulas, equivocarme en las cifras significativas al momento de reportar los datos, no comprendo bien las matemáticas, no identifico fórmulas específicas y 
poner la conversión al revés, obtuvieron la misma frecuencia (1). Es importante tener en cuenta que las categorías base no fueron las que presentaron las mayores frecuencias, emergiendo categorías que según la percepción de los estudiantes son errores relevantes al solucionar problemas.

Los resultados presentados anteriormente tienen que ver con lo que se denomina divorcio entre las definiciones de los conceptos matemáticos, su interpretación y su consecuente aplicación en la resolución de problemas, las afirmaciones realizadas por los estudiantes evidencian falencias en los procesos de análisis lógico matemático, habilidades que contribuyen sustancialmente al desarrollo de la competencia. Una consecuencia de cometer errores en la resolución de problemas es la generación de obstáculos conceptuales y procedimentales que desdibujan la concepción que los estudiantes apropian del procedimiento, complejizando el marco de referencia en el que se definen, dificultando el análisis, la interpretación y el procesamiento de la información, por ende, la correcta solución. Al respecto, Clark et al., (2017) demostraron que identificar errores es un proceso cognitivo de alto nivel que facilita encontrar la salida a las dificultades para superar los obstáculos, lo anterior sugiere que desarrollar ejercicios de autoevaluación en donde los estudiantes reconozcan los errores que se comenten, permite mejorar el procedimiento para resolver problemas y obtener mejores resultados.

\section{Aplicación del análisis dimensional - percepción de los profesores}

Para reconocer la aplicabilidad del análisis dimensional se identificaron las temáticas de química básica que requieren soporte matemático para solucionar problemas, a través de una nube de palabras (Fig. 2), el tamaño de la palabra indica la importancia que le otorgaron los profesores a las diferentes temáticas. Respecto a las temáticas que los profesores reconocieron con mayor frecuencia, propiedades de la materia, gases y estequiometría corresponden a las categorías base, adicionalmente, termodinámica y soluciones fueron categorías emergentes. Las temáticas mencionadas anteriormente, son ejes en el desarrollo de la asignatura química de primer semestre, siendo las bases conceptuales para poder desarrollar problemas disciplinares complejos en semestres superiores y durante el desarrollo profesional, tal como lo demostró Despuy et al., (2018) quienes relacionaron los temas mencionados anteriormente con los principales errores que cometen los estudiantes cuando resuelven problemas de química.

Por otra parte, medición y energía no son temas de exclusividad del área de química, sino que se aplican en diferentes áreas del conocimiento, demostrando que la resolución de problemas, usando la lógica matemática es ampliamente utilizada, por lo que el uso de estrategias que minimicen los errores, como los cometidos en los procesos de análisis dimensional es valioso para profesionales de diferentes contextos, al respecto Bois (2011) indica que en procesos experimentales de la biología, el análisis dimensional permite comprender fenómenos físicos.

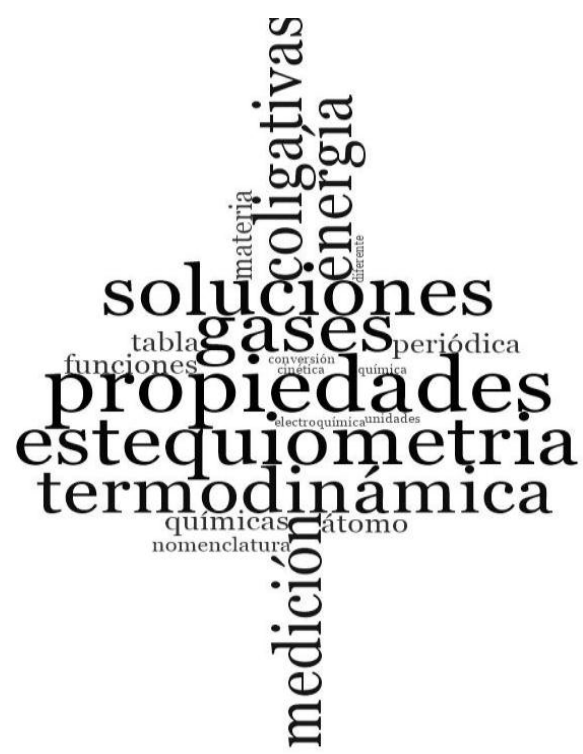

Fig. 2: Temáticas que requieren el uso de análisis dimensional

Finalmente, la aplicabilidad del análisis dimensional se evaluó en términos de diferentes actividades en las que se utilizan procedimientos matemáticos para resolver problemas, en la Fig. 3, se presentan los gráficos de codificación de datos, el eje x muestra los valores del atributo en un rango de 0 a 10, sobre la importancia que los profesores le asignan a cada actividad y en el eje y, el número de casos por aplicabilidad que le otorgan los participantes con base en la siguiente clasificación: A. Usar ejercicios aplicados, B. Usar 
problemas en contexto, C. Expresar magnitudes derivadas en términos de unidades fundamentales, D. Comprobar veracidad de las fórmulas, E. Deducir nuevas fórmulas, F. Hacer cálculos para resolver problemas, G. Agilizar la capacidad de análisis, H. Evidenciar la relación entre las unidades, I. Precisar lo que representan las expresiones, J. Promover el razonamiento dimensional.

a

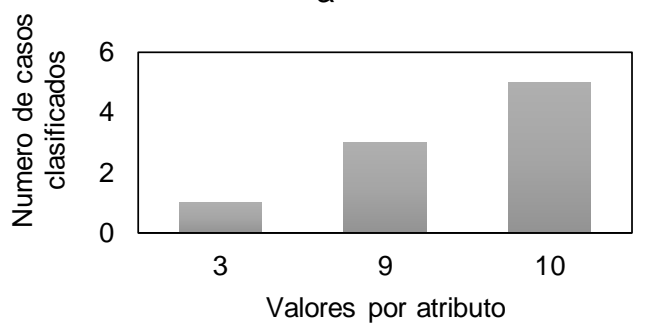

C

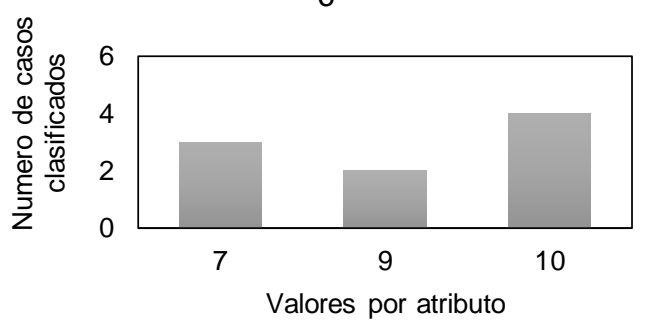

e

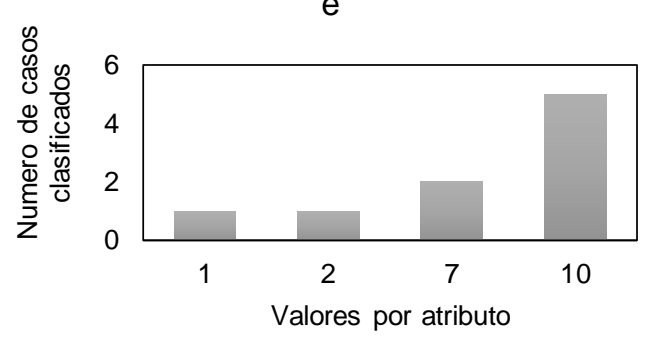

g
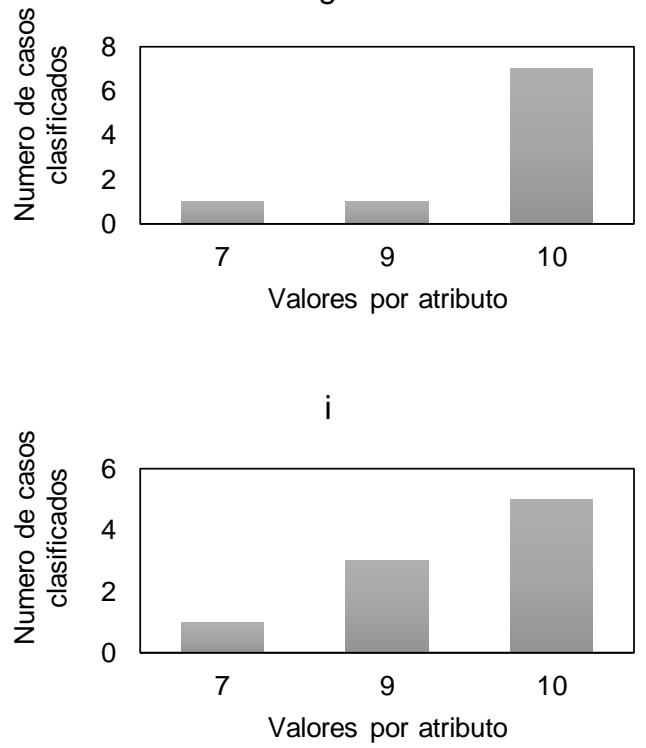

b

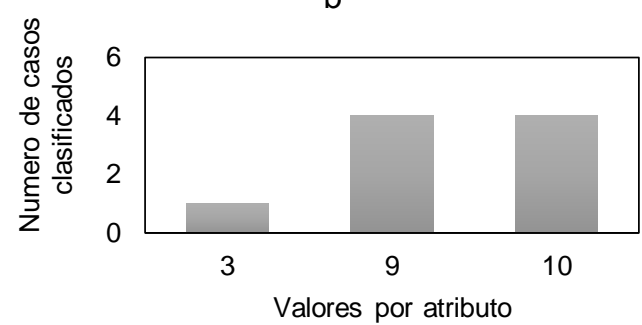

d

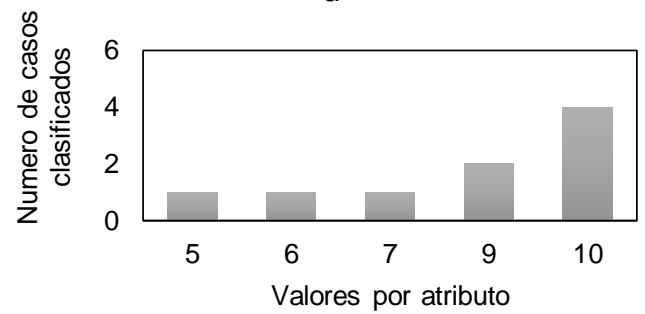

$f$

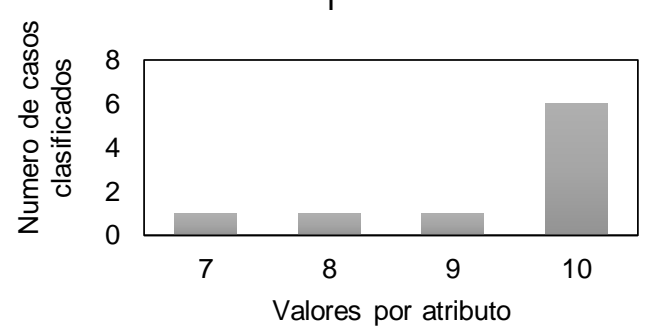

$\mathrm{h}$

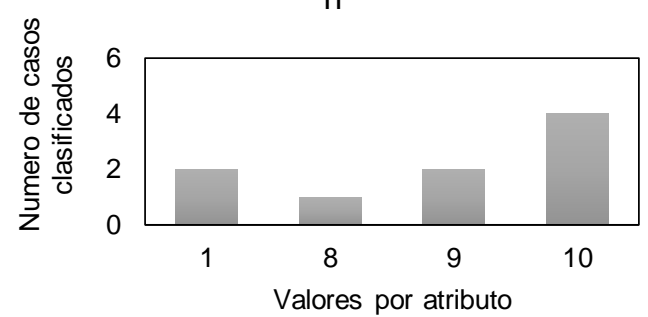

j

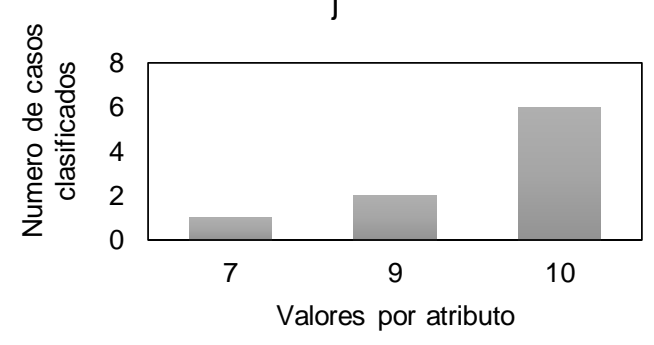

Fig. 3: Aplicabilidad que los profesores de química le atribuyen al análisis dimensional 
De los 9 profesores que participaron en el estudio, 7 utilizan el análisis dimensional principalmente para agilizar la capacidad de análisis (G), con respecto a lo anterior, Johnson (2021) sugiere que el cerebro cuando se expone a acciones novedosas realiza varias operaciones de pensamiento, que le permiten una mayor comprensión de los fenómenos. Por otro lado, 6 profesores manifestaron usar el análisis dimensional para hacer cálculos para resolver problemas (F), lo que se refuerza con las afirmaciones hechas por Pursell et al., (2017) referidas al análisis dimensional que puede ser utilizado como un enfoque educativo para mejorar la competencia matemática. Finalmente, 6 profesores lo usan para promover el razonamiento dimensional (J), ejercicio fundamental para la resolución de problemas tal como lo menciona Godino et al., (2002) quienes refieren que la reflexión en torno al tema de magnitudes y unidades es relevante dentro de cualquier enfoque matemático.

\section{CONCLUSIONES}

Con base en el análisis sobre la resolución de problemas en química, por parte de estudiantes y profesores universitarios, se concluye que:

1) Los estudiantes perciben como métodos para resolver problemas de química la regla de tres, la intuición, los factores de conversión, el uso de la tecnología a través de simuladores y videos, y los libros como nuevas formas para obtener información y construir conocimiento;

2) El análisis narrativo permite analizar los datos cualitativos descifrando categorías base y emergentes relacionadas con un tema de estudio;

3) El análisis dimensional permite resolver problemas en las cuales se requiere lógica matemática para su comprensión y abordaje, porque permite identificar apropiadamente las unidades y magnitudes, promoviendo la capacidad de análisis de los estudiantes y la competencia matemática;

4) Los profesores universitarios en el área de química fundamentan diversas estrategias pedagógicodidácticas, implementando el análisis dimensional, ratificando que es un método que promueve el razonamiento matemático y la competencia de Resolución de Problemas; y

5) La herramienta N-Vivo resulta útil para la calificación y la codificación de las categorías definidas en el análisis narrativo, permite descifrar las emergentes contabilizando las palabras registradas en una base de datos, asistiendo la organización que soporta la interpretación y el análisis cualitativo.

\section{AGRADECIMIENTOS}

Los autores agradecen el apoyo al Proyecto PG-04-2019: financiado por la Universidad Nacional Abierta y a Distancia - UNAD (Colombia) con alianza de las universidades nacionales La Sabana, Distrital Francisco José de Caldas, Universidad de Cundinamarca, la Universidad del Atlántico y la universidad argentina Nacional de la Patagonia Austral.

\section{REFERENCIAS}

Anilan, B., y Berber, A., Effect of conversion factor on problem solving and experience of teacher candidates, J. Res. Educ. Sci, ISSN: 2148-9955, 5(1), 118-133 (2019)

Bados, A., y García, E., Resolución de problemas, 3-31, Universitat de Barcelona, Barcelona, España (2014)

Bois, R., Adriao, O., y otros cuatro autores, Influence of process variables on foaming ability of surfactants: Experimental study and dimensional analysis, https://doi.org/10.1016/j.cherd.2020.10.021, Chem. Eng. Res. Des., 165, 40-50 (2021)

Bois, J.S., Dimensional analysis as a tool to introduce biology students to physical reasoning, https://doi.org/10.1016/j.bpj.2010.12.1974, Biophys. J., 100(3), Suplement 1, S1-640 (2011)

Bucheró, L. M., y Panche-Jardínes, R., Tareas docentes para contribuir a la competencia profesional de resolución de problemas de cálculo químico cuantitativo en la educación de adultos, Opuntia Brava, ISSN: 2222-081X,12(1), 21-36 (2020)

Castro, E. J., y Miranda, I., Experiencias desmotivacionales y motivacionales de estudiantes varones de Ingeniería para estudiar Matemáticas, El caso de la Universidad Andrés Bello en Santiago de Chile, http://dx.doi.org/10.4067/S071850062019000600083, Form. Univ., 12(6), 83-92 (2019)

Clark, L. A., Cuthbert, B., y otros tres autores, Three approaches to understanding and classifying mental disorder: ICD11, DSM-5, and the National Institute of Mental Health's Research Domain Criteria (RDoC), https://doi.org/10.1177/1529100617727266, Psychol. Sci. Public Interest, 18(2), 72-145 (2017) 
Despuy, G., Kern, S., Pacini, C., y Craveri, A., Estrategias interdisciplinarias para fortalecer el aprendizaje de la química a partir de dificultades en el desarrollo de competencias matemáticas, http://sedici.unlp.edu.ar/handle/10915/97658, IV Congreso Argentino de Ingeniería - X Congreso Argentino de Enseñanza de la Ingeniería, Córdoba, Argentina (2018)

Díaz, J. A., y Díaz, R., Los métodos de resolución de problemas y el desarrollo del pensamiento matemático, https://doi.org/10.1590/1980-4415v32n60a03, Bolema, 32(60), 57-74 (2018)

Dossey, J. A., Problem solving from a mathematical standpoint, The nature of problem solving: Using research to inspire 21st century learning, OECD Publishing, ISBN-10: 9264273891, 4, 59-72, Paris, Francia (2017)

Ergen, Y., ¿Does mathematics fool us? A study on fourth grade students non-routine maths problem solving skills, Issues Educ. Res., ISSN: 0313-7155, 30(3), 828-848 (2020)

Frodyma, M., Using elementary calculus and dimensional analysis to prepare students for physical chemistry, https://doi.org/10.1021/acs.jchemed.9b00807, J. Chem. Educ., 97(3), 741-748 (2020)

Gamboa, M. C., Vera-Monroy, S. P., Mejía-Camacho, A., y Romero, J. C., Resolución de problemas: Millennials y centennials, generaciones del análisis dimensional, http://doi.org/10.5281/4266566, Revolución en la Formación y la Capacitación para el Siglo XXI Vol. II. (33-40), Editorial Instituto Antioqueño de Investigación, Medellín, Colombia (2020)

Godino, J., Batanero, C., y Roa, R., Medida de magnitudes y su didáctica para maestros, Universidad de Granada, Granada, ISBN:84-932510-2-X, (635- 654) Granada, España (2002)

Gómez, B., Los ritos en la enseñanza de la regla de tres, José Mariano Vallejo, el matemático ilustrado. Una mirada desde la Educación Matemática, Primera edición, Servicio de Publicaciones de la Universidad de Córdoba, ISBN:847801-847-6, 47-69, Córdoba, Argentina (2006)

Gürsan, S., y Yazgan, Y., Non-Routine problem-solving skills of ninth grade students: An experimental study, https://doi.org/10.31805/acjes.632560, J. Educ. Sci., 4(1), 23-29 (2020)

Hernández, E., y González, S., Análisis de datos cualitativos a través del sistema de tablas y matrices en investigación educativa, https://doi.org/10.6018/reifop.435021, Rev. Electrón. Interuniv. Form. Profr., 23(3), 115-132 (2020)

Hernández, R., Fernández, C., y Baptista, P., Metodología de la investigación, 6ta edición, McGraw Hill, ISBN: 978-14562-2396-0, 154-155, México (2014)

Johnson, J., Learning from experience and performing learned actions are easy; novel actions, problem-solving, and calculation are hard, Chapter 10, Designing with the mind in mind, Third Edition, Morgan Kaufmann, ISBN 9780128182024, https://doi.org/10.1016/B978-0-12-818202-4.00010-6, 155-175, San Francisco, USA (2021)

Lira-Parada, P., Even, P., Biegler, L.T., y Bar, N., Implications of dimensional analysis in bioreactor models: Parameter estimation and identifiability, https://doi.org/10.1016/j.cej.2021.129220, Chem. Eng. Sci., 417 (2021)

Malaspina, U., Intuición, rigor y resolución de problemas de optimización, Rev. Latinoam. de Investig. en Mat. Educ., ISSN: 1665-2436,10(3), 365-399 (2007)

Mochón, S., Enseñanza del razonamiento proporcional y alternativas para el manejo de la regla de tres, RevEM, ISSN: 1665-5826, 24(1), 133-157 (2012)

Morgan, D., Learning in liminality. Student experiences of learning during a nursing study abroad journey: A hermeneutic phenomenological research study, http://dx.doi.org/10.1016/j.nedt.2017.09.017, Nurse Educ Today, 79, 204-209 (2019)

Morice-Singh, C., Indian Calculation: The rule of three-quite a story.... In let history into the mathematics classroom, 47-57, Springer, Cham, Suiza (2018)

Nizama, M., y Nizama, L.M., El enfoque cualitativo en la investigación jurídica, proyecto de investigación cualitativa y seminario de tesis, https://doi.org/10.24265/voxjuris.2020.v38n2.05, VOX JURI , 38(2), 69-90 (2020)

NVivo, qualitative data analysis software, QSR International Pty Ltd, Version NVivo (Release 1.0) / Nvivo 1.0 - March 18 (2020)

Pursell, D. P., Forlemu, N. Y., y Anagho, L. E., Mathematics competency for beginning chemistry students through dimensional analysis, https://doi.org/10.3928/01484834-20161219-05, J Nurs Educ., 56(1), 22-26 (2017)

Raviolo, A., Gennari, F., y Andrade, J. J., Integración conceptual en cursos de Química General, http://dx.doi.org/10.22201/fq.18708404e.2000.1.66479, Educ. Química, 11(1), 178-181 (2000)

Simamora, R. E., y Hasratuddin, S.S., Improving students' mathematical problem solving ability and self-efficacy through guided discovery learning in local culture context, https://doi.org/10.12973/iejme/3966, Electron. J. Math. Educ., 14(1), 61- $72(2019)$

Corbin, J., y Strauss, A., Basics of Qualitative Research: Techniques and Procedures for Developing Grounded Theory, 4rd ed., Sage Publications Inc, ISBN 9781412997461, 3-16, New Delhi, India, (2014) 